\title{
On the Determination of Flow Characteristics in Packed Column Chemical Reactors with Wall Effects
}

\author{
Thomas Z. Fahidy ${ }^{1}$ \\ ${ }^{1}$ Department of Chemical Engineering, University of Waterloo, Waterloo, ON, Canada \\ Correspondence: Thomas Z. Fahidy, Department of Chemical Engineering, University of Waterloo, Waterloo, ON, \\ N2L 3G1, Canada. Tel: 1-519-888-4567. E-mail: tfahidy@uwaterloo.ca
}

Received: September 9, 2013

Accepted: October 10, 2013 Online Published: October 21, 2013

doi:10.5539/ijc.v5n4p113

URL: http://dx.doi.org/10.5539/ijc.v5n4p113

\begin{abstract}
The estimation of the coefficient of equalization of velocities $(D)$, and the wall effect factor $(k)$ in a cylindrical flow-through packed bed reactor equipped with wire gauze packing is presented via properly truncated eigenfunction series subjected to regression analysis. The first numerical example indicates adequate fit of (posited) experimental data on the fraction of the total fluid in the wall layer by nonlinear regression, while the second example portrays the opposite situation where the same model fails to reliably represent the (posited) observation set. Regression upon linearization produces poor to absurd results.
\end{abstract}

Keywords: packed-bed reactor, regression analysis, Bessel functions $J_{0}$ and $J_{1}$

\section{Introduction}

An early characterization of liquid-flow distribution in a packed column with wall effect (Porter \& Jones, 1963) describes the two-dimensional flow pattern in terms of a "liquid spread factor" and a "wall factor" appearing in an infinite series which describes the radial distribution by Bessel functions of the first kind order zero $\left(J_{0}\right)$ and order one $\left(J_{1}\right)$, and the axial distribution by negative exponentials. Overestimation of the wall effect, tested experimentally in a six-inch diameter cylindrical column using half-inch Raschig rings, was ascribed to liquid channeling ignored by the model where the spreading mechanism of the liquid flow was assumed to be analogous to diffusion.

Adopting a somewhat different treatment of the theme (Jenson \& Jeffreys, 1977), the wall effect here is presented, upon some algebraic rearrangements, as

$$
F(z)=\frac{2 k}{b+2 k}+\frac{4 b k}{a} \sum_{n=1}^{\infty} \Phi_{n}(a, b, k) \exp \left(-\lambda_{n}^{2} D z\right) ; \Phi_{n} \equiv \frac{J_{l}\left(a \lambda_{n}\right)}{\lambda_{n}\left[b^{2}+2 b k+b^{2} k^{2} \lambda_{n}^{2}\right] J_{0}\left(b \lambda_{n}\right)}
$$

where $a$ is the radius of the concentric inlet tube at the top of the column, $b$ is the cylindrical column radius, $D$ is the dimensionless coefficient of equalization of velocities (a property of the packing and the fluid used), $F$ is the fraction of the total fluid in the wall layer, $k$ is the wall-effect factor, and $z$ is the dimensionless axial length. The summation represents an infinite series with alternating signs caused by the oscillatory behaviour of the $J_{0}(x)$ and the $J_{1}(x)$ functions with respect to the horizontal axis; this is an intrinsic property of the $J_{\mathrm{n}}(x)$ family ( $n$ is an integer) of Bessel functions (Watson, 1966). The eigenvalue set $\left\{\lambda_{n}\right\}$ contains the ascending roots of the algebraic equation

$$
k \lambda_{n} J_{0}\left(b \lambda_{n}\right)+J_{1}\left(b \lambda_{n}\right)=0
$$

The step-by-step derivation of Equation (1) is an elegant demonstration (Jenson \& Jeffreys, 1977) of converting an a-priori non-orthogonal set of eigenfunctions into an orthogonal set allowing the conventional application of orthogonality theory. It follows from Equation (1) that if the column is sufficiently long, $F(z \rightarrow \infty)=2 k /(b+2 k)$ is an acceptably close approximation to the true fluid flow fraction. The value of $z=0$ denotes the position where the fluid flow first extends continuously across the entire (horizontal) cross section.

When a packed column is used as a chemical reactor (with the packing material serving e.g. as a catalyst), its characteristic (D; k) parameters can be expected to differ from their counterparts in the simple flow-through case. Depending on the specifics of the reaction(s), certain physical properties in the reactor might also become 
sufficiently different to deny validity to Equation (1). Motivation for the current communication stems (i) from the desire for a relatively uncomplicated quantitative procedure to test the applicability of Equation (1) to any packed bed reactor with its geometry conforming, at least approximately, to the Jenson-Jeffreys formulation, and (ii) from the supposition that highly accurate and reliable (e.g. nanotechnology-based) fluid fraction versus axial position observations will be eventually available for practical utilization of the sequel.

The numerical illustrations demonstrate (i) a strong and a weak case for accepting Equation (1) as a good model of fluid fraction distribution, and (ii) the perils incurred when Equation (1) is linearized in search for an (apparently) effort-saving means of data fitting.

\section{Method}

In the first step, the wall effect factor is estimated by neglecting the eigenfunction expansion term in Equation (1), yielding

$$
k \cong \frac{b}{2} \cdot \frac{F(z)}{1-F(z)}, \quad \mathrm{z} \rightarrow \infty
$$

if $z$ is sufficiently large (i.e. $F(z)$ increases very little when $z$ is further increased). A least-squares estimate of the parameter $D$ can be obtained via conventional regression theory from the expression

$$
\frac{\partial}{\partial D} \sum_{j=1}^{J}\left[F_{e}\left(z_{j}\right)-F\left(z_{j}\right)\right]^{2}=0
$$

where $F_{\mathrm{e}}\left(z_{\mathrm{j}}\right) ; j=1, \ldots, J$ are the experimentally obtained flow ratios and $F\left(z_{\mathrm{j}}\right), j=1, \ldots, J$ are the flow ratios predicted by Equation (1). The $D$-estimate is given by solving

$$
\sum_{j=1}^{J}\left[F_{e}\left(z_{j}\right)-F\left(z_{j}\right)\right] \frac{\partial}{\partial D} F\left(z_{j}\right)=\varepsilon ; \frac{\partial}{\partial D} F\left(z_{j}\right)=-\frac{4 b k}{a} \sum_{n=1}^{\infty} \Phi_{n}(a, b . k)\left(\lambda_{n}^{2} z_{j}\right) \exp \left(-\lambda_{n}^{2} D z_{j}\right)
$$

where $\varepsilon$ is an arbitrarily assigned constant located near zero.

\section{Potential Applications to Chemical Reactions}

\subsection{Illustration No.1: Treatment of an Industrial Effluent}

An industrial effluent entering an experimental cylindrical flow reactor $(a=0.20 \mathrm{~m} ; b=0.80 \mathrm{~m})$ packed with a certain wire gauze is envisaged to leave it with its BOD (biochemical oxygen demand) reduced by chlorination within the range of $12-18 \mathrm{mg} / \mathrm{dm}^{3}$. The packing ensures a sufficiently large contact area, and it also acts as a suspended solids collector [the outflow is to contain less than $30 \mathrm{mg} / \mathrm{dm}^{3}$ suspended solids (Pletcher \& Walsh, 1990)]. Continuous flow-through operation is interrupted, when packing efficiency is no longer adequate, to install fresh packing and resume the normal treatment process. The experimental data contained in the first and second column of Table 1 are assumed, in the absence of currently available information, for the sole purpose of numerical demonstration of the method of attack. 
Table 1. Analysis of regression of Equation (1) in Section 3.1. $a=0.2 \mathrm{~m}, b=0.8 \mathrm{~m}, k=0.058 \mathrm{~m}, 4 b k / a=0.928 \mathrm{~m}$

\begin{tabular}{ccccc}
\hline Dimensionless & \multicolumn{4}{c}{ Fraction of total fluid at the wall, per cent } \\
\cline { 2 - 5 } axial length, $z$ & Experimental $^{(1)}$ & Equation (1); $n=1$ & Equation $(1) ; n=1,2$ & Equation $(1) ; n=1,2,3$ \\
\hline 0.2 & 5.38 & 4.26 & 3.99 & 3.98 \\
0.3 & 8.48 & 8.02 & 7.25 & 7.25 \\
0.4 & 9.35 & 10.11 & 9.44 & 9.44 \\
0.5 & 9.71 & 11.27 & 10.78 & 10.78 \\
0.6 & 10.92 & 11.91 & 11.57 & 11.57 \\
0.7 & 11.85 & 12.27 & 12.04 & 12.04 \\
0.8 & 12.12 & 12.46 & 12.32 & 12.32 \\
0.9 & 12.48 & 12.57 & 12.48 & 12.48 \\
\hline
\end{tabular}

(1) posited for the sake of illustration.

Eigenvalues: $\lambda_{1}=4.490, \lambda_{2}=8.225, \lambda_{3}=11.987,[\lambda]=\mathrm{m}$.

Eigenfunctions: $\Phi_{1}=-1.3266, \Phi_{2}=0.2926, \Phi_{3}=-0.1936,[\Phi]=\mathrm{m}^{-1}$.

Coefficient of determination $R^{2}=0.924 ; 0.932 ; 0.931$.

Dimensionless coefficient of velocity equalization $D=0.292 ; 0.263 ; 0.263 ; \varepsilon \leq 10^{-5}$.

Residuals distribution $\left[F_{\mathrm{e}}(z)-F(\mathrm{z})\right.$ versus $\left.F(\mathrm{z})\right]$ : quasi-random.

\subsection{Illustration No.2: An Electro-Organic Reaction System}

While, in principle, many reaction systems could qualify for the purpose, the electrolytic oxidation of aqueous phenol solutions to hydroquinone HQN (known also as p-quinone, 1,4-benzoquinone, and 1,4 dihydroxybenzene) in the presence of dilute sulphuric acid) is employed as an example. The reaction is assumed to be studied in an exploratory flow-through reactor, whose wire gauze packing is coated with finely dispersed lead oxide particles acting as anode. Vertical cathode rods are inserted in a proper pattern allowing prior supposition of a multiple undivided-cell structure. For the purpose of the current subject matter it is sufficient to indicate the simplified process mechanism (Rifi \& Covitz, 1974) amongst e.g. the thirty four pre-1975 reports on its electro-organic synthesis (Swann \& Alkire, 1980).Accordingly, phenol first reacts with water to produce quinone, free protons and free electrons, whose subsequent interaction yields the desired product HQN and molecular hydrogen. Reoxidation of HQN is avoided by simultaneous/competitive adsorption of phenol and quinone at the anode. The first and second column of Table 2 show the experimental data posited by the same reasoning as in Section 3.1.

Table 2. Analysis of regression of Equation (1) in Section 3.2. $a=0.1 \mathrm{~m}, b=0.5 \mathrm{~m}, k=0.038 \mathrm{~m}, 4 b k / a=0.760 \mathrm{~m}$

\begin{tabular}{cccc}
\hline \multirow{2}{*}{ Dimensionless axial length, $z$} & \multicolumn{3}{c}{ Fraction of total fluid at the wall, per cent } \\
\cline { 2 - 4 } & Experimental $^{(1)}$ & Equation (1) $\mathrm{n}=1$ & Equation (1) $\mathrm{n}=1,2$ \\
\hline 0.05 & 8.20 & 7.50 & 7.56 \\
0.10 & 10.30 & 12.37 & 12.37 \\
0.15 & 11.60 & 13.07 & 13.07 \\
0.20 & 12.30 & 13.17 & 13.17 \\
0.25 & 12.90 & 13.19 & 13.19 \\
0.30 & 12.95 & 13.19 & 13.19 \\
0.35 & 12.98 & 13.19 & 13.19 \\
0.40 & 12.99 & 13.19 & 13.19 \\
0.45 & 13.05 & 13.19 & 13.19 \\
0.50 & 13.12 & 13.19 & 13.19 \\
\hline
\end{tabular}

(1) posited for the sake of illustration.

Eigenvalues: $\lambda_{1}=7.15, \lambda_{2}=13.13,[\lambda]=\mathrm{m}$.

Eigenfunctions: $\Phi_{1}=-0.3919 ; \Phi_{2}=0.4960,[\Phi]=\mathrm{m}^{-1}$.

Coefficient of determination: $R^{2}=0.662 ; 0.664$.

Dimensionless coefficient of velocity equalization $\mathrm{D}=0.756 ; 0.735 \varepsilon \leq 10^{-7} ; 5 \times 10^{-4}$. 


\section{Analysis and Discussion}

The conventional coefficient of determination (or squared correlation coefficient), written for the context of this paper, as

$$
R^{2}=1-\frac{\sum_{j=1}^{J}\left[F_{e}\left(z_{j}\right)-F\left(z_{j}\right)\right]^{2}}{\sum_{j=1}^{J}\left[F_{e}\left(z_{j}\right)-F_{m}\right]^{2}}
$$

expresses the extent to which a regression explains the variation in the experimental data [or, put otherwise, gives "...the proportion of the variation of the dependent variable which is taken up by fitting the regression line..." (Porkess, 2005)]. Regressions with $R^{2} \geq 0.9$ are generally considered to be a good fit of data, if the distribution of the residuals $F_{\mathrm{e}}\left(z_{\mathrm{j}}\right)-F\left(z_{\mathrm{j}}\right)$ shows at least a reasonable tendency toward randomness (e.g., Montgomery, Runger, \& Hubele, 2001). Appendix 1 summarizes certain tests concerning hypotheses about large-experimental-set based (population) values in face of the set of experimental observations considered as a sample from said population.

\subsection{The Packed Bed Reactor in Section 3.1}

Table 1 demonstrates a close fit of data by Equation (1), and it also indicates that treatment based on the leading eigenvalue alone is quite adequate from a statistical point of view. Nevertheless, a process analyst might prefer the results based on both the first and second eigenvalues and accept $D=0.263$ rather than 0.292 ; consideration of higher eigenvalues would be redundant (except perhaps at $z$ values very close to zero).

\subsection{The HQN Reactor in Section 3.2}

As depicted in Table 2, Equation (1) is at best weakly adequate for correlating the $F$ versus $z$ relationship. Reasons for this weakness are not immediately obvious, but flow channeling, adhesion to cathode rods and (partial) local clogging of the gauze anodes e.g. would be likely candidates.

\subsection{Regression on the Linearized Form of Equation (1): The Case Against It}

Linearization of Equation (1) by considering merely the leading eigenvalue and the leading eigenfunction (the only possibility for linear regression), might appear to be tempting in order to simplify numerical encumbrance often accompanying nonlinear regressions. Under such conditions an auxiliary function $Y_{1}$ can be defined as

$$
Y_{I}(z) \equiv \ln \frac{(2 k /(b+2 k)-F(z)}{(4 b k / a)\left(-\Phi_{I}\right)}=-\lambda_{I}^{2} D z
$$

Equation (7) implies, in principle, a linear regression $y_{1}=b_{0}+b_{1} z$ on the experimental $F$ versus $z$ data strings. The slope of this regression yields a parameter estimate

$$
D \approx \frac{-b_{1}}{\lambda^{2}}
$$

by neglecting $\mathrm{b}_{0}$ on the basis of failing to reject the null hypothesis $\mathrm{H}_{0}: \beta_{0}=0$ (Appendix $2 ; \beta_{0}$ is the population intercept).

While Appendix 1 and Appendix 2 along with Table 3 and Table 4 indicate sufficient quantitative reasons for avoiding linearization in the two illustrations, linearization itself is objectionable in principle, however manipulatively convenient it might appear to be. If $Y=f(X)$ denotes transformation of a random variable $X$ to random variable $Y$, the variance relationship $V(\mathrm{Y})=(\mathrm{d} Y / \mathrm{d} X)^{2} V(X)$ finally leads to $V(Y)=V(X) \exp (-2 Y)$ in the case of linearization, hence $V(Y)$ changes from observation to observation. The weighted linear least squares method (e.g. Mickley, Sherwood, \& Reed, 1957) employing sample variance $s_{\mathrm{Y}}^{2}$, in place of the (unknown) population variance $V(Y)$, usually different at each observation, offers no particular advantages over regressions involving nonlinear least squares. 
Table 3. Linearized regression of Equation (1) in Section 3.1. $a=0.2 \mathrm{~m}, b=0.8 \mathrm{~m}, k=0.058 \mathrm{~m}, 4 b k / a=0.928 \mathrm{~m}$

\begin{tabular}{|c|c|c|c|}
\hline \multirow{2}{*}{ Dimensionless axial length, $z$} & \multicolumn{2}{|c|}{$-y_{1}(z)$} & \multirow{2}{*}{$F_{1}(z)^{(2)}$} \\
\hline & Experimental & Equation $(7)^{(1)}$ & \\
\hline 0.2 & 1.3193 & 1.1760 & 1.80 \\
\hline 0.3 & 1.8631 & 1.6859 & 5.83 \\
\hline 0.4 & 2.0993 & 2.0968 & 9.37 \\
\hline 0.5 & 2.2127 & 2.5577 & 9.97 \\
\hline 0.6 & 2.7291 & 3.0186 & 10.98 \\
\hline 0.7 & 3.4621 & 3.4795 & 11.62 \\
\hline 0.8 & 3.8389 & 3.9404 & 12.02 \\
\hline 0.9 & 4.7809 & 4.4013 & 12.28 \\
\hline
\end{tabular}

Table 4. Assessment of linearization failure in Section 3.2

Equation (7): $Y_{1}(z)=\ln [0.44179-3.3484 F(z)]=-51.1225 D z$.

For $z>0.10, Y_{1}(z)$ is a complex quantity inasmuch as the argument of the logarithmic function is negative.

The "brute force" estimate of $D=0.175$ obtained from $Y_{1}(0.05)=-1.7884 ; Y_{1}(0.10)=-2.2354$ yields

$F_{1}(z)=13.19-39.30 \exp (-8.9464 z)$ per cent with absurd negative estimates $F_{1}(0.05)=-11.94$ and

$F_{1}(0.10)=-2.87$ per cent. Consequently, $R^{2}=1-346.46 / 49.610=-5.98$ (Equation 6 ) is equally absurd.

\subsection{Computational Aspects}

A key element in the determination of parameters $(D ; k)$ is the eigenvalue set $\left\{\lambda_{n}\right\}$. The fortuitously rapid growth of the eigenvalues usually forestalls the necessity of finding eigenvalues beyond a small number (Tables 1-3 indicate the impunity of neglecting all but the first and second eigenvalue in the illustrations). Apart from mathematical/statistical software, such as Microsoft EXCEL and Press et al. (1986, 1992), extensive tabulations of the $J_{0}$ and the $J_{1}$ functions can be found in Abramowitz and Stegun (1965), Jahnke and Emde (1945), Tuma (1979, 1989), G. Korn and Th. Korn (1968) and the standard mathematical tables published regularly by CRC Press. Watson (1966) provides a very thorough theoretical background for the application of Bessel functions. Approximations via Chebyschev polynomials carrying 20-digit decimals (Luke, 1975) exemplify the degree of available numerical precision.

It is worth noting (although in a negative sense) that the experimental data in Section 3.2 can be correlated "almost perfectly" by the cubic regression $F(z)=0.0556+0.6206 z-1.6965 z^{2}+1.5198 z^{3}$ with $R^{2} \approx 1.0$. It would be useless for the purposes of this paper, since the parameters $(D ; k)$, intrinsic properties of Equation (1), could not be extracted from it.

\section{Final Remarks and Conclusion}

The ultimate purpose of model identification is to allow the process analyst to determine how process/design parameters are to be modified, if necessary, for desired performance. When useful for finding appropriate $(D ; k)$ values for an a-priori stipulated flow fraction distribution (such as in Section 3.1), Equation (1) would be an attractive tool of reactor design and performance analysis. In opposite cases (such as in Section 3.2), a different approach to formulating the flow mechanism is warranted. The discussion of such "follow-up" steps is beyond the scope of this paper.

\section{Acknowledgments}

The University of Waterloo and the Natural Sciences and Engineering Research Council (NSERC) of Canada have provided facilities for this work. 


\section{References}

Abramowitz, M., \& Stegun, I. A. (1965). Handbook of Mathematical Functions with Formulas, Graphs, and Mathematical Tables (pp. 390, 392). New York, NY: Dover.

Arnold, S. F. (1990). Mathematical Statistics (p. 420). Englewood Cliffs, NJ: Prentice Hall.

Beyer, W. H. (1968a). Handbook of Tables for Probability and Statistics (2nd ed., pp. 125-134). Boca Raton, FL: CRC Press.

Beyer, W. H. (1968b). Handbook of Tables for Probability and Statistics (2nd ed., pp. 282-285). Boca Raton, FL: CRC Press.

Jahnke, E., \& Emde, F. (1945). Tables of Functions with Formulas and Curves (4th ed., pp. 156-163). New York, NY: Dover.

Jenson, V. G., \& Jeffreys, G. V. (1977). Mathematical Methods in Chemical Engineering (2nd ed., pp. 285-290) London, UK: Academic Press.

Korn, G. A., \& Korn, Th. M. (1968). Mathematical Handbook for Scientists and Engineers (2nd.ed., pp. 1036-1042). New York, NY: McGraw Hill.

Lindley, D. V., \& Scott, W. F. (1984b). New Cambridge Statistical Tables (pp. 42-45). Cambridge, UK: Cambridge University Press.

Lindley, D. V., \& Scott, W. F. (1984a). New Cambridge Statistical Tables (pp. 34-35). Cambridge, UK: Cambridge University Press.

Luke, Y. L. (1975). Handbook of Functions and Their Applications (pp. 322-324). New York, NY: Academic Press.

Mickley, H. S., Sherwood, T. K., \& Reed, Ch. E. (1957). Applied Mathematics in Chemical Engineering (2nd ed., pp. 97-99). New York, NY: McGraw Hill.

Montgomery, D. C., Runger, G. C., \& Hubele, N. F. (2001). Engineering Statistics (2nd ed., pp. 336-337). New York, NY: Wiley and Sons.

Neter, J., Wasserman, W., \& Kutner, M. H. (1990). Applied Linear Statistical Methods (3rd ed., pp. 71-73). Boston, MA: R. D. Irwin.

Pletcher, D., \& Walsh, F. C. (1990). Industrial Electrochemistry (2nd ed., pp. 331-333). London, UK: Chapman and Hall.

Porkess, R. (2005). Collins Dictionary of Statistics (2nd ed., pp. 49-51). Glasgow, UK: Harper Collins.

Porter, K. E., \& Jones, M. C. (1963). A Theoretical Prediction of Liquid Distribution in a Packed Column with Wall Effect. Transactions of the Institution of Chemical Engineering, 41, 240-247.

Press, W. H., Flannery, B. P., Teukolsky, S. A., \& Vetterling, W. T. (1986). Numerical Recipes (pp. 170-176). Cambridge, UK: Cambridge University Press.

Press, W. H., Teukolsky, S. A., Vetterling, W. T., \& Flannery, B. P. (1992). Numerical Recipes in C (2nd ed., pp. 230-236). Cambridge, UK: Cambridge University Press.

Rifi, M. R., \& Covitz, F. H. (1974). Introduction to Organic Electrochemistry (pp. 157-158). New York, NY: Marcel Dekker.

Snedecor, G. W., \& Cochran, W. G. (1989). Statistical Methods (8th ed., pp. 187-190). Ames, Iowa: Iowa State University Press.

Swann, Jr., Sh., \& Alkire, R. (1980). Bibliography of Electro-Organic Syntheses 1801-1975 (p. 526). Baltimore, MD: Port City Press.

Tuma, J. J. (1979). Engineering Mathematics Handbook (p. 194). New York, NY: McGraw Hill.

Tuma, J. J. (1989). Handbook of Numerical Calculations in Engineering (p. 382). New York, NY: McGraw Hill.

Watson, G. N. (1966). A Treatise on the Theory of Bessel Functions (pp. 38-47). Cambridge, UK: Cambridge University Press. 


\section{Appendix 1. Statistical Testing of Hypotheses Involving the Correlation Coefficient of a Linear Regression}

Given the correlation coefficient $r=\sqrt{ } R^{2}$ of a linear regression $y=b_{0}+b_{1} x$ obtained on the basis of a sample, originating from a large sample/population with true regression $Y=\beta_{0}+\beta_{1} z$. The null hypothesis $\mathrm{H}_{0}: \rho=0$, where $\rho$ is the large-sample/population correlation coefficient can be tested by the T-distribution statistic ( e.g., Snedecor \& Cochran, 1989; Arnold, 1990):

$$
t=r \frac{(n-2)^{1 / 2}}{\left(1-R^{2}\right)^{1 / 2}}
$$

when compared to the critical value $t(\alpha ; n-2)$ at a chosen significance level $\alpha$, tabulated for certain values of $n$ and $\alpha$ in statistical texts and handbooks. A second procedure, called Fisher's z-transformation, uses the standardized approximately normal statistic

$$
z_{0}=(n-3)^{1 / 2}\left(\frac{1}{2} \ln \frac{1+r}{1-r}-\frac{1}{2} \ln \frac{1+\rho}{1-\rho}\right)
$$

to test the null hypothesis of setting an arbitrary value, i.e., $\mathrm{H}_{0}: \rho=\rho_{0}$ (equivalent in this context to the null hypothesis $\mathrm{H}_{0}: \rho \neq 0$ ) and finding the error incurred if $\mathrm{H}_{0}$ were rejected from (standardized) normal distribution tables e.g., Beyer, 1968a; Lindley \& Scott, 1984a).

In Section $3.1 t \approx 15.96$ obtained via Equation (A.1) for $y_{1}$ indicates that $\rho=0$ is untenable. In Section 3.2, since Equation (A.1) yields $t \approx 3.67$, rejection of $\mathrm{H}_{0}: \rho=0$ would carry only a $0.5 \%$ error $[t(8 ; 0005) \approx 3.36]$. Applying Equation (A.2) to the correlation coefficients $R^{2} \approx 0.98$ in Section 3.1 is too high for postulating a substantially lower $\rho$ below 0.99 , while material here cannot be applied to Section 3.2 on account of the failure of linear regression.

\section{Appendix 2. Statistical Testing of Hypotheses Involving the Intercept of a Linear Regression}

Given the linear regression $y=b_{0}+b_{1} x$ obtained on the basis of a sample, originating from a large-sample/population with true regression $Y=\beta_{0}+\beta_{1} x$. To test the null hypothesis $\mathrm{H}_{0}: \beta_{0}=0$, the T-distribution statistic (e.g., Neter, Wasserman, \& Kutner, 1990)

$$
t=\frac{b_{0} s_{x}}{s} \cdot \frac{[n(n-1)]^{1 / 2}}{\left[\sum_{i=1}^{n} x_{i}^{2}\right]^{1 / 2}} ; s^{2} \equiv \frac{(n-1)\left(s_{y}^{2}-b_{1}^{2} s_{x}^{2}\right)}{n-2}
$$

is computed; $n$ is the sample size, $s_{\mathrm{x}}{ }^{2}$ the variance of the set of independent variables $\left\{x_{\mathrm{n}}\right\}$, and $s_{\mathrm{y}}{ }^{2}$ the variance of the set of dependent variables $\left\{y_{\mathrm{n}}\right\}$. If the value of $t$ in Equation (A.3) exceeds the critical value $\mathrm{t}(\alpha, n-2)$ at a chosen significance level $\alpha$ tabulated for certain values of $\alpha$ and $n$ (e.g., Beyer, 1968b; Lindley \& Scott, 1984b), then $\mathrm{H}_{0}$ is rejected (at the chosen significance level $\alpha$ ) and $\beta_{0}>0$ or $\beta_{0}<0$ is accepted, depending on the sign of $b_{0}$. In Section $3.1, s_{\mathrm{x}}{ }^{2}=0.05998, s_{\mathrm{y}}{ }^{2}=1.3360 ; b_{0}=-0.2532 ; b_{1}=-4.6090 ; n=8$; Equation (A.3) yields $t \approx-1.02$, hence rejection of $\mathrm{H}_{0}$ carries a large error of about $20 \%[t(0.2 ; 6) \approx-0.91$ on the negative half-plane of the T-distribution]. Similarly to Appendix 1, material in Section 3.2 does not qualify for the test in Equation (A.3).

\section{Copyrights}

Copyright for this article is retained by the author(s), with first publication rights granted to the journal.

This is an open-access article distributed under the terms and conditions of the Creative Commons Attribution license (http://creativecommons.org/licenses/by/3.0/). 